\title{
Modelling and Optimisation of Industrial Absorption Processes: An EC Collaborative Research Project
}

\author{
P. Seferlis ${ }^{\text {a, }}$, N. Dalaouti ${ }^{a}$, E. Y. Kenig ${ }^{b}$, B. Huepen ${ }^{\text {b }}$, P. Patil ${ }^{c}$, M. Jobson ${ }^{c}$, J. Klemes ${ }^{c}$, \\ P. Proios ${ }^{\mathrm{d}}$, M. C. Georgiadis ${ }^{\mathrm{d}}$. E. N. Pistikopoulos ${ }^{\mathrm{d}}$, S. Singare ${ }^{\mathrm{e}}$, C. S. Bildea ${ }^{\mathrm{e}}$, \\ J. Grievink ${ }^{\mathrm{e}}$, P. J. T. Verheijen ${ }^{\mathrm{e}}$, M. Hostrup ${ }^{\mathrm{f}}$, P. Harper ${ }^{\mathrm{f}}$, G. Vlachopoulos ${ }^{\mathrm{g}}$, \\ C. Kerasidis ${ }^{\mathrm{g}}$, J. Katsanevakis ${ }^{\mathrm{h}}$, D. Constantinidis ${ }^{\mathrm{h}}$, P. Stehlik ${ }^{\mathrm{i}}$, and G. Fernholz ${ }^{\mathrm{j}}$ \\ ${ }^{a}$ CERTH - Chemical Process Engineering Research Institute (CPERI), PO Box 361, \\ 57001 Thermi Thessaloniki, Greece \\ ${ }^{\mathrm{b}}$ Department of Biochemical and Chemical Engineering, University of Dortmund, \\ PO Box 44221, Emil-Figge Str. 70, 44227, Dortmund, Germany \\ ${ }^{\mathrm{c}}$ Centre for Process Integration, CEAS, The University of Manchester PO Box 88, \\ Sackville St., Manchester, United Kingdom \\ ${ }^{\mathrm{d}}$ Centre for Process Systems Engineering, Department of Chemical Engineering, \\ Imperial College London, London SW7 2AZ, United Kingdom \\ ${ }^{\mathrm{e}}$ Delft Chem Tech, Delft University of Technology, Julianalaan 136, 2628 BL, Delft, \\ The Netherlands \\ ${ }^{\mathrm{f}}$ CapSolva - Integrated Process Solutions ApS, Kronprinsessegade 46 E, DK-1306 \\ Kbh. K, Copenhagen, Denmark \\ ${ }^{g}$ Phosphoric Fertilizers Industry S.A., P.O. Box 10183, 54110 Thessaloniki, Greece \\ ${ }^{\mathrm{h}}$ ESTIA Consulting S.A., Makrigianni 61, 57001 Thermi Thessaloniki, Greece \\ ${ }^{\mathrm{i}}$ Institute of Process and Environmental Engineering, Technical University of Brno, \\ Technicka 2, 61669 Brno, Czech Republic \\ ${ }^{\mathrm{j}}$ Process Systems Enterprise Ltd, Bridge Studios, 107a Hammersmith Bridge Road, \\ London, W6 9DA, United Kingdom
}

\begin{abstract}
This work summarises the research and technological achievements of the EC funded research project OPT-ABSO. The major innovation of the present project is the development of a fully integrated set of modelling, simulation, and optimisation methodologies, and computer-aided tools for the design, synthesis, control, and efficient operation of sustainable industrial absorption processes. The aim of cost, waste and energy reduction and the development of realistic operating strategies for practical implementation are facilitated through an interactive software framework.
\end{abstract}

Keywords: Absorption, Modelling, Synthesis, Optimisation, CAPE tools

\footnotetext{
*Author/s to whom correspondence should be addressed: seferlis@cperi.certh.gr
} 


\section{Introduction}

Reactive absorption is attracting the interest of the chemical industry as it provides an efficient alternative for the recovery of significant pollutants and useful compounds from industrial gas streams in relatively mild operating conditions. Considering the environmental impact of reactive absorption that necessitates the operation under tight specifications it is imperative that reliable and accurate modelling tools become available for the design and operation simulation, optimisation and analysis.

The current work summarises the developments in the modelling technology of reactive absorption achieved in OPT-ABSO a European collaborative project. Furthermore, a brief description of the integrated tools that cover the synthesis, optimisation and heat integration studies tailored to the specific needs of reactive absorption is provided.

\section{Modelling of Reactive Absorption Processes}

Design, optimisation and analysis of reactive absorption processes require the use of reliable process models that predict with the desired degree of accuracy the interactions between the multiple phenomena occurring within the column. Tray and packed absorption columns can be described as a sequence of equivalent stages. These stages are modelled using a rigorous rate-based model, which incorporates the mass and heat transfer mechanisms and the complex reaction schemes involved in industrial reactive absorption operations (Kenig et al., 2001). The model is based on the two-film approach that considers mass and heat transfer resistance concentrated within two film regions adjacent to the interface, where phase equilibrium is assumed to hold (see Figure 1). The models cover a wide range of absorption processes including electrolyte systems and reacting systems in both the liquid and gas phases. Furthermore, the dynamic dimension of the models enables the prediction of the transient behaviour and the evaluation of the control system performance during operation.

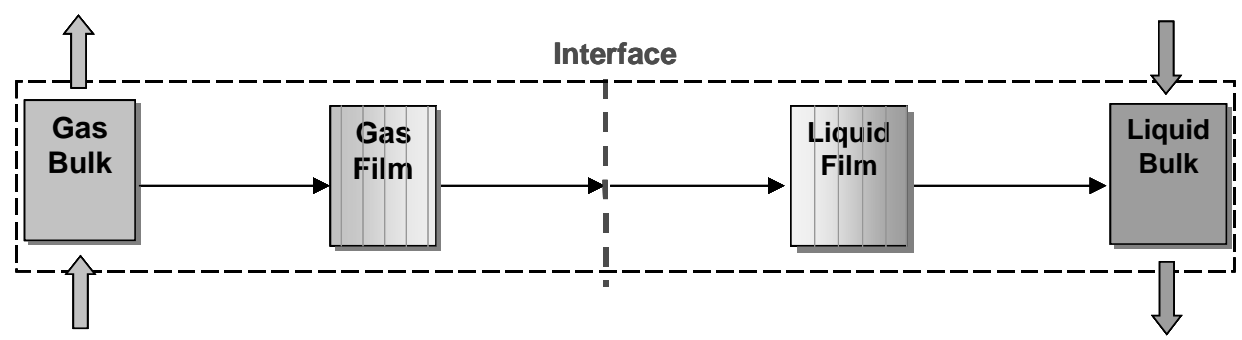

Figure 1. Schematic of the rate-based stage model

Process design and real time applications however, may require the use of simplified or reduced-order models that enable quick calculations to verify the feasibility of the separation. Techniques varying from orthogonal collocation on finite elements (OCFE) to balanced residualisation (Singare et al., 2003) are employed to construct reducedorder models of variable resolution and controlled accuracy (Figure 2). Most notably, OCFE formulation allows for a continuous representation of a staged column in a compact form. Simplified models based on the Kremser group method allowed for preliminary design studies of the gas sweetening absorption-desorption flowsheets with 
multiple feed and recycle streams (Patil and Jobson, 2004).

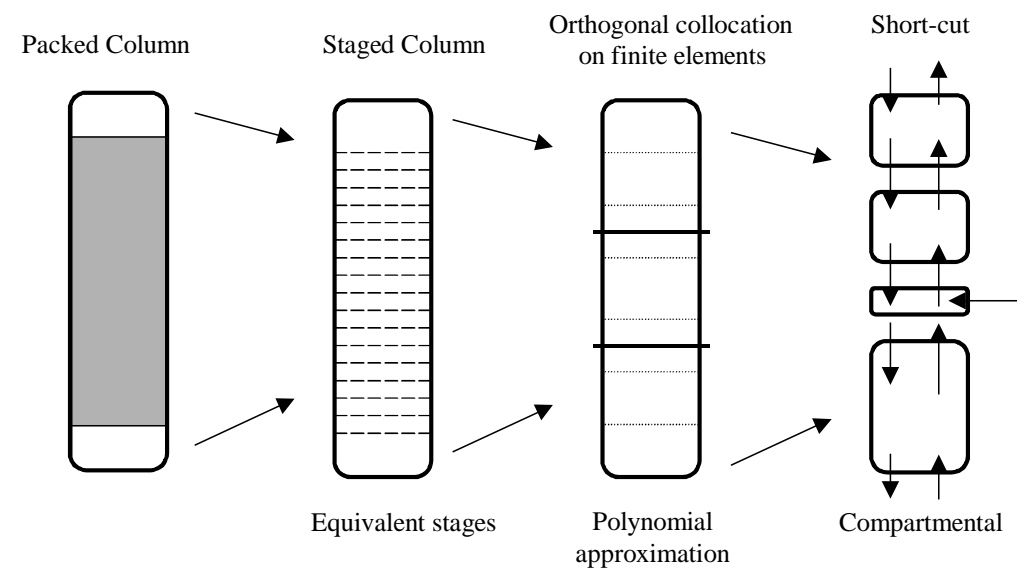

Figure 2.Modelling approximations

\section{Synthesis, Design, Control and Optimisation}

\subsection{Synthesis of absorption sequences}

The Generalized Modular Framework (GMF) is a flexible methodology for process synthesis based upon superstructure optimisation techniques. The GMF method produces a compact mathematical formulation, which can be subdivided into components, consisted of a Structural Model, and a Physical Model (Figure 3). The Structural Model acts as the generator for the process structural alternatives, while the function of the Physical Model is the evaluation of feasibility and optimality of each structural alternative generated based upon a valid representation of the underlying physical phenomena. The synthesis methodology allows the optimal design of process flowsheets while achieving the tight separation targets under model and process related uncertainties.

GMF Mass/Heat Module

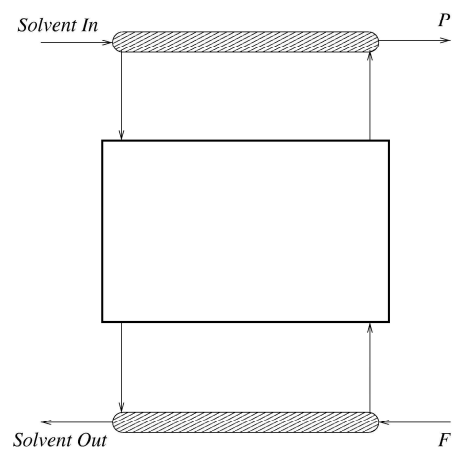

GMF/OC Mass/Heat Module

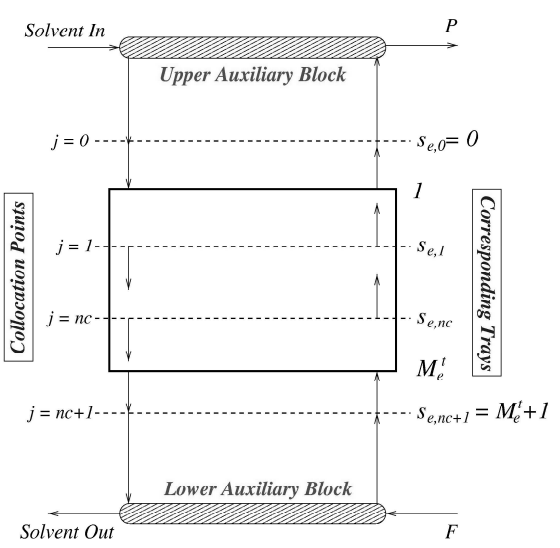

Figure 3. GMF module and its discretised (orthogonal collocation) equivalence. 


\subsection{Controllability analysis of reactive absorption units}

Controllability analysis examines the impact of process design on the performance of the control system; an issue of paramount importance considering the stringent constraints that absorption units have to satisfy (e.g., as pollution prevention units in $\mathrm{NO}_{\mathrm{x}}$ removal or waste incineration gas cleaning). A number of instruments have become available for the process characterisation and controllability analysis of reactive absorption units ranging from interaction analysis to static and dynamic disturbance rejection properties. Reduced-order linear approximations and nonlinear models in combination with advanced optimisation and linear algebra techniques are utilised in the evaluation, rank ordering and screening of alternative design configurations for the absorption columns.

\section{Validation and Industrial Case Studies}

Validation was performed with experimental data from pilot plant columns for coke oven gas purification (electrolyte system) and $\mathrm{NO}_{\mathrm{x}}$ removal and two industrial columns for nitric acid production (see Figure 4). Good match of the model predictions to the actual data was possible without any tuning of the model parameters (Figure 5). The effect of design decisions and particularly the location of the side feed and recycle streams, the cooling policy, and the nominal operating conditions on the dynamic behaviour of the column were investigated. Design decisions influence the dynamics of the column and consequently the performance of the control system. The analysis focused on the ability of the column and the implemented control system to alleviate the effects of disturbances in the temperature and concentration of the inlet gas stream to the product streams (Dalaouti and Seferlis, 2005). The evaluation criteria involved the steady-state impact of the disturbances on the controlled and manipulated variables (static controllability index) and the characteristics of the dynamic response (Table 1).

Heat integration studies of new plants and retrofit design of industrial absorption units using pinch-analysis revealed an area of great potential for essential energy saving opportunities. Optimisation-based techniques have been applied on two industrial cases (nitric and sulphuric acid production flowsheets) with proposed design modifications that reduce significantly the energy requirements of the existing plants.

In flue gas cleaning (e.g., waste incineration gases) an optimised Venturi scrubber (Figure 6) was designed that generated strong turbulence due to the increased velocity and allowed intensive mixing of the liquid agent (e.g., water solution of $\mathrm{NaOH}$ ) with the off-gases. A novel equipment design the "O-element" (Figure 7) resulted in significant reduction of the pressure drop for the gas stream and enhanced absorption efficiency.

Table 1. Static controllability index for simultaneous disturbance on the inlet gas temperature and $\mathrm{NO}_{x}$ content (preferred config. 20-55)

\begin{tabular}{ccccccc}
\hline \multicolumn{2}{c}{ Disturbance variation (\%) } & 5 & 10 & 15 & 20 & 25 \\
\hline $\begin{array}{c}\text { Side stream } \\
\text { position }\end{array}$ & $\begin{array}{c}\text { Recycle stream } \\
\text { position }\end{array}$ & \multicolumn{5}{c}{ Static controllability index } \\
\hline 20 & 55 & 0.529 & 0.780 & 1.030 & 1.227 & 1.445 \\
20 & 60 & 0.525 & 0.773 & 1.016 & 1.270 & 1.494 \\
30 & 55 & 0.580 & 0.884 & 1.152 & 1.423 & 1.614 \\
30 & 60 & 0.588 & 0.870 & 1.132 & 1.405 & 1.670 \\
\hline
\end{tabular}




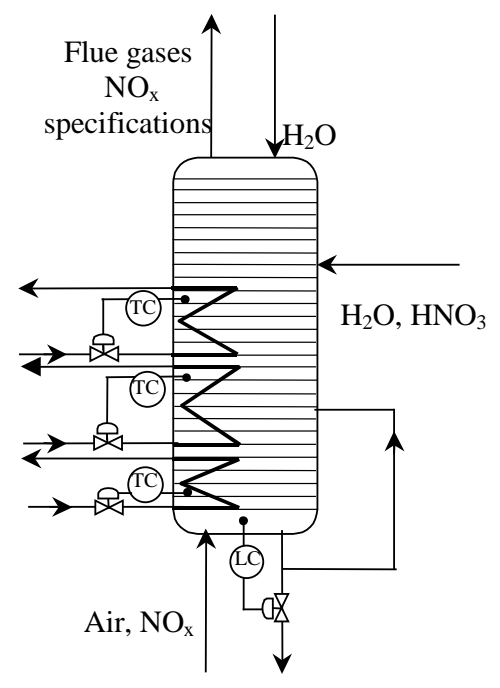

Figure 4. Reactive absorption for $\mathrm{HNO}_{3}$ production

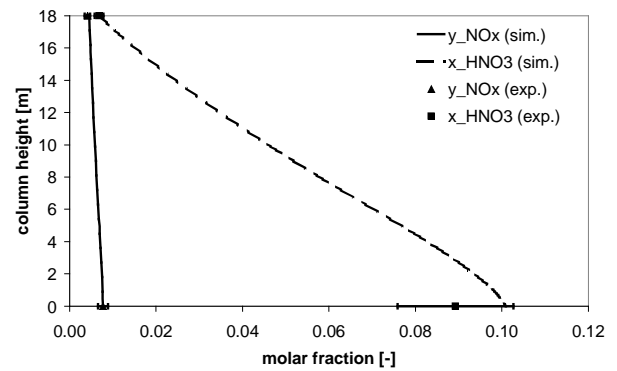

Figure 5.Rate-based model validation for the absorber in the $\mathrm{HNO}_{3}$ production

\section{Software Framework}

The entire suite of models and tools for absorption processes has become available through a software prototype framework (note: version for evaluation and testing is available, www.opt-abso.org). The software prototype comprises a set of interconnected components for input specification, execution management, output visualisation, and review, a set of model libraries (model defining sets of equations) and model server components (for the evaluation of the model equations) and a set of activity execution components (e.g. simulation, optimisation, calculation or analysis).

Excel $^{\circledR}$ is the platform for the input, output and execution management communication, while the basic simulation and optimisation engine is gPROMS $^{\circledR}$ (Process Systems Enterprise, 2004). Additional third party software components can be linked to the prototype to perform the required analysis tasks (e.g., MATLAB).

The main modelling options included in the software prototype: (i) rate-based model for steady-state and dynamic simulation, (ii) OCFE with rate-based balances for design and operation optimisation and controllability analysis, (iii) OCFE with equilibrium balances for column synthesis (iv) simplified process model with Murphree efficiencies for synthesis, and (v) reduced state space models for controllability analysis. In addition, a tool for solvent selection in natural absorption processes was developed. In principle, given the information on desired properties relevant to the separation system (e.g., solubility) and the type of compound (e.g., aromatic, cyclic, number of C, ester, alcohol) a set of compounds is derived that satisfy the separation specifications. The tool's ability to handle mixtures of compounds enables greater flexibility and makes it suitable for problems of industrial relevance. The key activities that are available in the OPT-ABSO software prototype are summarised in Table 2. 


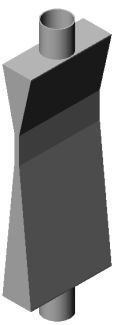

Figure 6. Venturi scrubber for flue gas cleaning

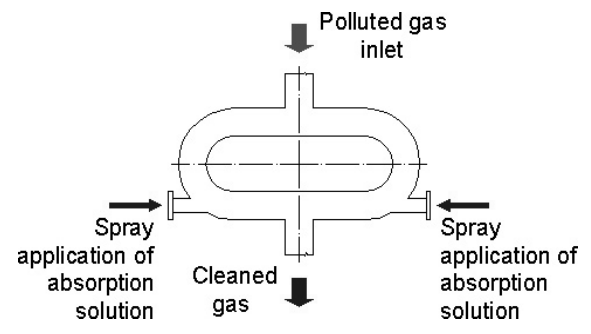

Figure 7. O-Element for flue gas cleaning

Table 2. Tools and features of the OPT-ABSO software prototype.

\begin{tabular}{lcccccc}
\hline Reactive absorption & \multicolumn{2}{c}{ Simulation } & Synthesis & Optimisation & $\begin{array}{c}\text { Controllability } \\
\text { analysis }\end{array}$ & $\begin{array}{c}\text { Heat } \\
\text { integration }\end{array}$ \\
\hline & steady-state & dynamic & & & & \\
\hline $\begin{array}{l}\text { Nitric acid production } \\
\text { Sulphuric acid }\end{array}$ & $\mathrm{x}$ & $\mathrm{x}$ & $\mathrm{x}$ & $\mathrm{x}$ & $\mathrm{x}$ & $\mathrm{x}$ \\
$\begin{array}{l}\text { production } \\
\text { Coke-oven gas }\end{array}$ & $\mathrm{x}$ & $\mathrm{x}$ & & & & \\
Gas sweetening & $\mathrm{x}$ & & $\mathrm{x}$ & & $\mathrm{x}$ \\
$\mathrm{CO}_{2}$ absorption & $\mathrm{x}$ & $\mathrm{x}$ & $\mathrm{x}$ & & & \\
\hline
\end{tabular}

\section{Conclusions}

The comprehensive software framework of integrated tools provides the state-of-the-art technology for the synthesis, design, and optimisation of reactive absorption units. Issues that may need further exploration are concentrated in the developments of correlations for mass and heat transfer coefficients for trays and packings with specific geometry through the analysis of experimental results, and the detailed study of system hydraulics (e.g., effect of liquid phase maldistribution). The application of the models and tools to biotechnological systems shows great potential for future research.

\section{Acknowledgments}

The financial support of the European Commission (Growth programme G1RD-CT2001-00649) is gratefully acknowledged.

\section{References}

Dalaouti N., and P. Seferlis, 2005, J. Cleaner Prod., (in press).

Kenig E.Y., R. Schneider, and A. Gorak, 2001, Chem. Eng. Sci., 343, 56.

Patil P., and M. Jobson, 2004, ESCAPE-14, 457.

Process Systems Enterprise, 2004, gPROMS v.2.3.1. User's Guide.

Proios P, and E. N. Pistikopoulos, 2003, ESCAPE-13, 263.

Singare S., C. S. Bildea, and J. Grievink, 2003, ESCAPE-13, 929. 